\title{
Establishment of Empirical Formula of Drilling Force for Stainless Steel Micro Hole
}

\author{
Zhiyong wang, Longfei Cao, Zhufeng zhang \\ Mechanical Engineering College, Yanshan University, Qinhuangdao, 066004, China \\ email: wangzy@ysu.edu.cn
}

Keywords: Micro Hole; Micro Drill; Drilling Force; Empirical Formula

\begin{abstract}
To solve the actual production problem that micro drill easily prone to break during micro drilling process, the process for drilling micro hole of $0.08 \mathrm{~mm}$ on the $316 \mathrm{~L}$ stainless steel was conducted and the main factors are analyzed, which demonstrate that the excessive torque is the main reason for breaking of micro drill. Therefore, the regression analysis for the drilling force under different drilling parameters is analyzed and the empirical formula of the drilling force is obtained. Meanwhile, the significance and the accuracy are also analyzed. According to the obtained empirical formula, the optimum drilling parameters during drilling process of stainless steel is established, and its validation is further confirmed in the actual production process.
\end{abstract}

\section{Introduction}

The micro hole is gradually widely used in the high technical field of aviation, spaceflight and micro-electronics and its machine process is always a difficult point in the machine building industry. As compared to the non-traditional machining, the machine drilling is more applicable for micro drilling[1]. However, the main problem is that the micro drill easily prone to break during micro drilling process.

For the problem that mentioned above, the influence of main structural parameters of micro drill on the cutting force and static and dynamic characteristics of the micro drill, was analyzed by Anish paul[2] from the point of geometric construction of micro drill and the optimizing of the micro drill was conducted. The dynamic signal was monitored in the drilling process to predict the breaking of the micro drill studied by Spdat Karabay[3]. The drilling parameters were optimized by genetic algorithm studied by Man Sheel Cheong[4], and the axial force and torque of the micro drill were decreased during the drilling process to prevent the breaking of the micro drill. In the any method mentioned above, the drilling force in the drilling process should be known[5] [6]. However, due to the structural difference between the micro drill and the twist drill, the traditional empirical formula of the drilling force is not applicable to this working condition. Hence, the empirical formula of the drilling force of the micro drill should be established and the drilling parameters should be optimized to decrease the broken rate of the micro drill in the drilling process.

The process for drilling micro hole of $0.08 \mathrm{~mm}$ on the $316 \mathrm{~L}$ stainless steel was taken as an example. The theoretical calculation and the morphology of the broken micro drill in the actual drilling are combined to obtain the main reason for the breaking of the micro drill. Based on the above analysis, the regression analysis is analyzed to obtain the empirical formula of the drilling force and the extent of the impact of the drilling parameter on the breaking of the micro drill, which can provide a basis for the optimization of the drilling parameter.

\section{The analysis of the reason for breaking of the micro drill}

Theoretical analysis for breaking. The root of the spiral groove of the micro drill is a weak part in the actual production and the breaking behavior of the tool is always located here. In theory, the micro drill in only bear the axial force and drilling torque in the drilling process. The process for drilling micro hole of $0.08 \mathrm{~mm}$ on the 316L stainless steel was simulated by Deform-3D, and the results of axial drilling force and torque, with a revolving speed $\mathrm{n}$, of $40000 \mathrm{r} / \mathrm{min}$, and a feed $\mathrm{f}$, of $0.004 \mathrm{~mm} / \mathrm{r}$, are shown in Figure1. After data processing, the mean axial force $\mathrm{F}$ of $0.4 \mathrm{~N}$ and mean 
drilling torque $\mathrm{M}$ of $0.03 \mathrm{~N} \cdot \mathrm{mm}$ are obtained.

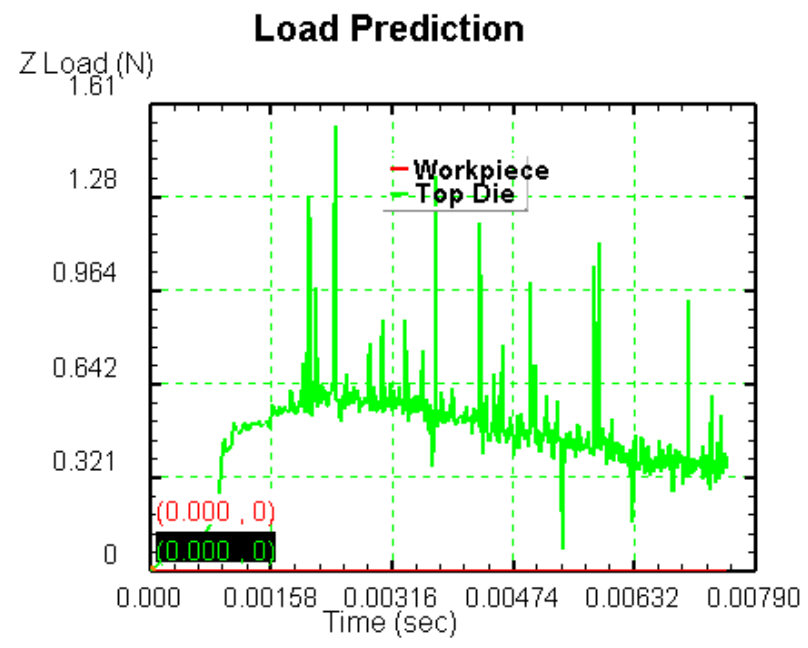

(a) Axial drilling force

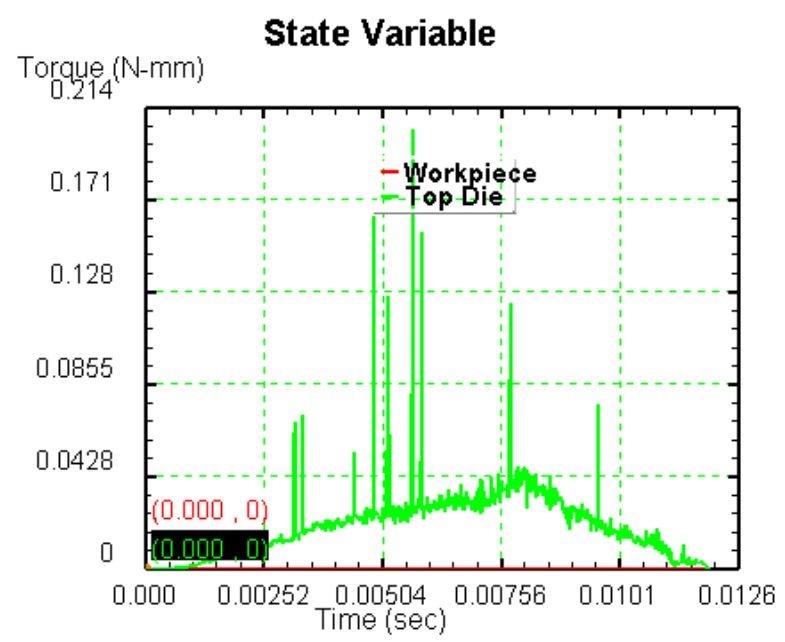

(b) Drilling torque

Fig.1. Drilling force

During the drilling process, the stress in the arbitrary cross section is mainly composed of the axial normal stress caused by axial drilling force and torsion shear stress caused by drilling torque. The former is of uniform distribution in the arbitrary cross section, while the latter has a maximum value at the edge of the cross section. Combined with the diameter of the micro drill of $0.08 \mathrm{~mm}$, the axial normal stress $\sigma$ in cross section is $80 \mathrm{MPa}$, and the torque shear stress $\tau_{\text {max }}$ in cross section is $300 \mathrm{MPa}$. According to the fourth strength theory, the resulting stress is expressed as below:

$$
\sigma_{r 4}=\sqrt{\sigma^{2}+3 \tau_{\max }^{2}}=525 M P a
$$

Formula (1) can be written

$$
\sigma_{r 4}=\sqrt{\sigma^{2}+3 \tau_{\max }^{2}}=\sigma \sqrt{1+\left[\frac{\sqrt{3} \tau_{\max }}{\sigma}\right]^{2}}
$$

If the $\mathrm{k}$ is assumed as:

$$
k=\frac{\sqrt{3} \tau_{\max }}{\sigma}=6.5
$$

Where $\mathrm{k}$ represents a influence factor that the shear stress impact on the resulting stress compared to the normal stress.

According to the fourth strength theory, the influence factor $\mathrm{k}$ is 6.5 . The $\mathrm{k}$ is greater than 1 and it demonstrates the influence of the shear stress due to drilling torque on the resulting stress is a litter greater than that of normal force. Hence, it can be concluded that the excessive torque is the main reason for breaking of micro drill in the ideal drilling condition.

Practical reason analysis for breaking. The fracture appearance can be observed under the scanning electron microscope are shown in Figure2. The broken part is at the root of the spiral groove and the crack is initially generated at this groove. The surface of the fracture appearance is an oblique section, which is of 45 degree compared to the cross section, and it is basically similar compared with the fracture appearance of the broken micro drill under the pure torque load. As a consequence, it can be predicted that the main reason for the breaking of the micro drill at the part of spiral groove in the drilling process is the excessive torque. To prevent the breaking of the micro drill, the drilling torque should be reduced and it can be achieved by two methods. One is the modification of the geometrical parameters of the micro drill; the other is the modification of the technological parameter. Because of the small dimension of the micro drill, the modification of the geometrical parameters of the micro drill cannot be achieved by coping that usually applied on the 
twist drill of the stock size, and thus only the second method can be used.

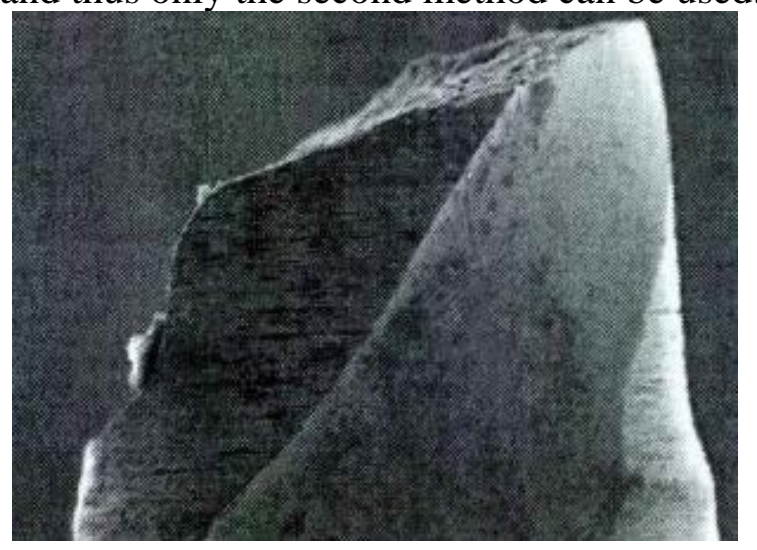

Fig.2. Fracture Appearance

\section{Establishment of the empirical formula of the drilling torques}

Regression analysis. The regression analysis method is adopted, and the feed $\mathrm{f}$ and the revolving speed of the main shaft are termed as two variables. Combined with the drilling process simulation by Deform-3D, the results of drilling torque are shown in Table 1. For the drilling process using diameter $\mathrm{d}$ of carbide drill, of $0.08 \mathrm{~mm}$, on the 316L stainless steel, the empirical formula of the drilling torque $\mathrm{M}$ is fitted.

It is assumed that the empirical formula of the drilling torque is expressed as below:

$$
M=A f^{a} n^{b}
$$

The linear treatment is done for the formula (4), i.e., the napierian logarithm is made for both sides of of the formula and the formula is obtained:

$$
\lg M=\lg A+a \lg f+b \lg n
$$

To be easy to calculate, it is assumed that $y=\operatorname{lgM}, c=\lg A, x_{1}=\operatorname{lgf}, x_{2}=\lg$, the equation of binary linear regression of the drilling torque can be expressed as below:

$$
y=c+a x_{1}+b x_{2}
$$

The formula is a linear equation, namely that there is a linear relationship between the independent variables of $x_{1} 、 x_{2}$ and dependent variable $y$, and this formula is the linear equation of the empirical formula of the drilling torque.

The napierian logarithm is made for the torque data and the results is shown in Table 1.

Table 1 Drilling torque with different drilling parameters

\begin{tabular}{cccccc}
\hline $\begin{array}{l}\text { Revolving speed of } \\
\text { main shaft / } / \mathrm{min}\end{array}$ & Feed / mm/r & Torque / N.mm & $\operatorname{lnv}$ & $\operatorname{lnf}$ & $\ln M$ \\
\hline 50000 & 0.001 & 0.015 & 4.698 & -3 & -1.824 \\
50000 & 0.005 & 0.047 & 4.698 & -2.301 & -1.328 \\
50000 & 0.01 & 0.082 & 4.698 & -2 & -1.086 \\
40000 & 0.001 & 0.014 & 4.602 & -3 & -1.854 \\
40000 & 0.005 & 0.046 & 4.602 & -2.301 & -1.337 \\
40000 & 0.01 & 0.077 & 4.602 & -2 & -1.114 \\
30000 & 0.001 & 0.013 & 4.477 & -3 & -1.886 \\
30000 & 0.005 & 0.042 & 4.477 & -2.301 & -1.377 \\
30000 & 0.01 & 0.069 & 4.477 & -2 & -1.161 \\
\hline
\end{tabular}

The regression equation used by multi-pole regression method: 


$$
Y=-1.2518+0.7 .82 x_{1}+0.3524 x_{2}
$$

Then the empirical formula for the drilling torque is expressed as below:

$$
M=0.056 n^{0.35} f^{0.74}
$$

For the tool under these conditions, the formula 8 is the empirical formula for the drilling torque.

Significance and accuracy analysis. To confirm the validity of the obtained empirical formula, the significance and accuracy analysis are done for the formula 7 and the analysis results are shown in Table 2.

Table 2 Significance analysis

\begin{tabular}{cccccc}
\hline & quadratic sum & Degree of freedom & variance & F & significance \\
\hline Regression & 0.853968 & 2 & 0.426984 & & \\
Residual error & 0.000349 & 6 & 0.000058 & 7331.4 & $6.84 \mathrm{E}-11$ \\
Total & 0.854318 & 8 & & & \\
\hline
\end{tabular}

Because the $\mathrm{F}$ is greater than the $\mathrm{Fa}(2,6)$, i.e. $\mathrm{F}>\mathrm{F}_{\mathrm{a}}(2,6)=10.92$, the regression equation is at a significance level of 6.84E-11.

For the significance level of $6.84 \mathrm{E}-11$, it can be verified that the regression quadratic sum is much greater than residual error quadratic sum, and the regression is highly significant, namely that the fitting drilling torque empirical formula is accurate.

\section{Establishment of the drilling parameter and the actual validation}

The drilling torque empirical formula is simulated by MATLAB, and the rule that the influence of two variables, including revolving speed of main shaft $(10000 \sim 60000 \mathrm{r} / \mathrm{min})$ and feed $(0.001 \sim$ $0.01 \mathrm{~mm} / \mathrm{r}$ ) on the drilling torque, is obtained. The result of response surface is shown in Figure3.

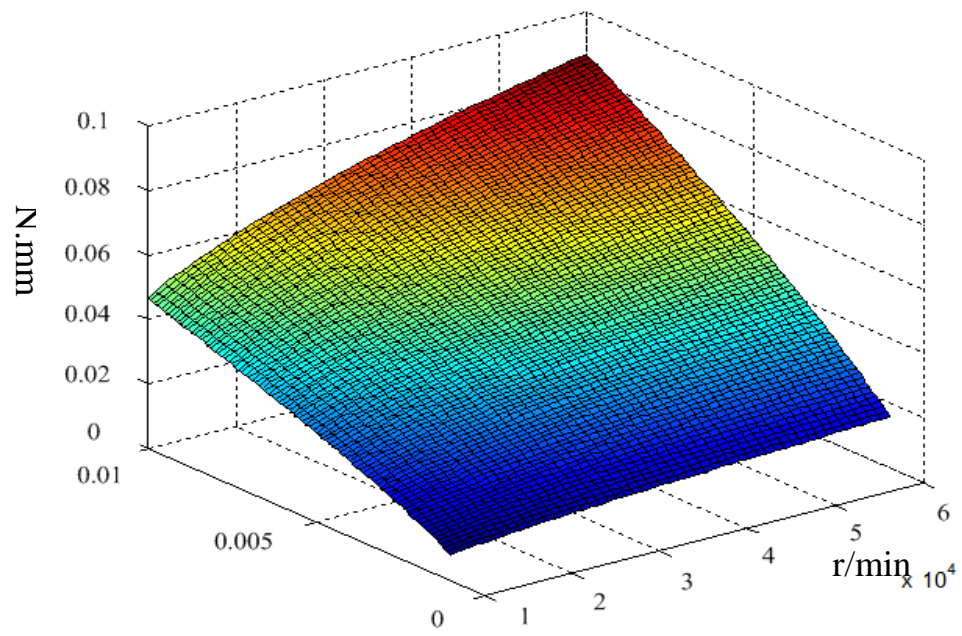

Fig.3. The response surface of the drilling torque

It can be seen from the Figure3. that the torque has a minimum value corresponding to the low revolving speed and low feed. The drilling torque exhibits a gradually increased trend with the increase of the revolving speed of the main shaft and the feed. Compared to the influence of these two variables on the drilling torque, the varied feed has a significant effect; especially the increase of feed has a greater effect than that of increase of revolving speed of the main shaft. Therefore, the decreased feed is firstly considered during the course of choosing proper drilling parameters.

In the actual production for processing of valve port of some gas flow meter, according to the results of empirical formula of drilling torque and simulated torque response, the revolving speed of $58000 \mathrm{r} / \mathrm{min}$ and feed of $0.005 \mathrm{~mm} / \mathrm{r}$ are selected. For a micro drill, it can averagely and continuously drill 530 holes in the actual production, which can drill additional 180 holes compared to that of the traditional processing method with the working condition of the revolving speed of $40000 \mathrm{r} / \mathrm{min}$ and feed of $0.01 \mathrm{~mm} / \mathrm{r}$. The actual production verifies that the empirical formula of the drilling torque is 
correct, and the empirical formula can provide a guiding function for the actual production.

\section{Conclusion}

The excessive torque is the main reason for breaking of micro drill in the micro drilling process. As a consequence, the empirical formula for the micro drilling torque can be established on the basis of this analysis. Then the analysis can be done for this empirical formula for the micro drilling torque, and it can be obtained that the varied feed has a great effect on the drilling torque. From the point of reducing drilling force, the decreased feed and high revolving speed are firstly considered during the course of choosing proper drilling parameters.

\section{References}

[1] Tang ying, Cheng duo. Micro-Hole High-Speed Drilling Process for Aluminum Alloy[J]. AeronauticalManufacturingTechnology, 20115 46-50.

[2] Anish P. Chisel edge and cutting lip shape optimization for improved twist drill point design[J]. International Journal of Machine Tool \& Manufacture, 2005 25(4-5) 421-431.

[3] Sedat K. Analysis of drill dynamo meter with octagonal ring type transducers[J]. Materials and Design, 2007 28(2) 673-685

[4] Man S C. Identification and control for micro-drilling productivity[J]. International Journal of Machine Tool \& Manufacture, 1999 39(10) 1539-1561.

[5] Tang Ying, Ma Hekun, Wu Quan. Simulation of Thrust Force and Torque for Micro Hole Drilling[J].Tool Engineering,2009 43(6) 61-66

[6] Duck W K. Tool life improvement by peck drilling and thrust force monitoring during[J]. International Journal of Machine Tool \& Manufacture, 2009,49 246-255 